\title{
Reliable detection of directional couplings using rank statistics
}

\author{
Daniel Chicharro ${ }^{1}$ and Ralph G. Andrzejak ${ }^{1}$ \\ ${ }^{1}$ Universitat Pompeu Fabra, Department of Information and Communication Technologies, Barcelona, Spain
}

(Dated: June 15, 2009)

\begin{abstract}
To detect directional couplings from time series various measures based on distances in reconstructed state spaces were introduced. These measures can, however, be biased by asymmetries in the dynamics' structure, noise color, or noise level, which are ubiquitous in experimental signals. Using theoretical reasoning and results from model systems we identify the various sources of bias and show that most of them can be eliminated by an appropriate normalization. We furthermore diminish the remaining biases by introducing a measure based on ranks of distances. This rank-based measure outperforms existing distance-based measures concerning both sensitivity and specificity for directional couplings. Therefore, our findings are relevant for a reliable detection of directional couplings from experimental signals.
\end{abstract}

PACS numbers: 05.45.Tp, 05.45.Xt, 05.45.-a

\section{INTRODUCTION}

A detection of directional couplings between two dynamical systems $X$ and $Y$ from the analysis of signals measured from them is key to an understanding of many dynamics in nature. Assuming $X$ and $Y$ to be linear stochastic Gaussian processes, the concept of Granger causality [1] can be implemented using linear regression or autoregressive modelling. This approach can be combined with graphical models [2] and directional couplings can be studied in a multivariate context [3]. Furthermore, different approaches were proposed to detect directional couplings from nonlinear or nonGaussian processes (see [4] for a review), including nonlinear Granger causality [5], transfer entropy [6], phase dynamics measures [7], as well as various measures evaluating distances of conditioned neighbors in reconstructed state spaces $[8,9]$. Applications to experimental time series in many scientific fields are found in different branches of physics, engineering, atmospheric sciences, neuroscience, biology or economy. There is, however, evidence that all these approaches can be affected by asymmetries in the dynamics' structure or noise level (e.g. [6, 9-11]). Here, we focus on the effect of these asymmetries on existing state space approaches. We furthermore introduce a rank-based measure and illustrate its robustness against such asymmetries. For that purpose we analyze the Lorenz dynamics as a representative example of a model system. For such a model system we can adjust the coupling strength and noise level which allows us to evaluate the sensitivity and specificity of the measures under controlled conditions. We focus on one representative example to provide a thorough statistical assessment and theoretical reasoning for the different sources of biases arising at different coupling strengths and asymmetries in the dynamics' structure, noise color, or noise level.

These state space approaches mainly consider the case where $X$ and $Y$ are assumed to be separate deterministic dynamics which both exhibit an independent self-sustained motion. It is further assumed that if there is a coupling it is uni-directional and too weak to induce a synchronized motion. Importantly, the dynamics are assumed to be stationary for the time window under investigation. It is not assumed that a meaningful instantaneous phase can be extracted from the signals. Under these assumptions, directional couplings can in principle be detected by quantifying the probability with which close states of the driven dynamics are mapped to close states of the driving dynamics $[8,9,13,14]$.

\section{METHODS}

Various distance-based approaches were proposed to evaluate this fundamental criterion (e.g. [8, 15-18]). For scalar time series $x_{i}$ and $y_{i}\left(i=1, \ldots N^{*}\right)$ simultaneously measured from $X$ and $Y$, respectively, the dynamics are reconstructed using delay coordinates $\mathbf{x}_{i}=\left(x_{i}, \ldots x_{i-(m-1) \tau}\right), \mathbf{y}_{i}=\left(y_{i}, \ldots y_{i-(m-1) \tau}\right)$ with embedding dimension $m$ and delay $\tau\left(i=1, \ldots N=N^{*}-(m-1) \tau\right)[16]$. By $v_{i, j}$ and $w_{i, j}(j=1, \ldots k)$ we denote the time indices of the $k$ spatially nearest neighbors of $\mathbf{x}_{i}$ and $\mathbf{y}_{i}$, respectively. From these we exclude temporal neighbors within $\left|v_{i, j}-i\right| \leq W$ and $\left|w_{i, j}-i\right| \leq W[16]$. For each $\mathbf{x}_{i}$, the mean squared Euclidean distance to its $k$ nearest neighbors is $R_{i}^{k}(X)=\frac{1}{k} \sum_{j=1}^{k}\left|\mathbf{x}_{i}-\mathbf{x}_{v_{i, j}}\right|^{2}$, and the Y-conditioned distance is: $R_{i}^{k}(X \mid Y)=\frac{1}{k} \sum_{j=1}^{k}\left|\mathbf{x}_{i}-\mathbf{x}_{w_{i, j}}\right|^{2}$. The mean 
distance to all other points is $R_{i}(X)=\frac{1}{N-1} \sum_{j=1, j \neq i}^{N}\left|\mathbf{x}_{i}-\mathbf{x}_{j}\right|^{2}$ [24] [25]. Based on these distances one can define:

$$
\begin{array}{r}
S(X \mid Y)=\frac{1}{N} \sum_{i=1}^{N} \frac{R_{i}^{k}(X)}{R_{i}^{k}(X \mid Y)} \\
H(X \mid Y)=\frac{1}{N} \sum_{i=1}^{N} \log \frac{R_{i}(X)}{R_{i}^{k}(X \mid Y)} \\
M(X \mid Y)=\frac{1}{N} \sum_{i=1}^{N} \frac{R_{i}(X)-R_{i}^{k}(X \mid Y)}{R_{i}(X)-R_{i}^{k}(X)}
\end{array}
$$

Eqs. (1 -2 ) were defined in [8]. Eq. (3 ) was derived in [17] from a measure proposed in [15] and independently from [17] in [16]. We here propose the following rank-based statistics: For each $\mathbf{x}_{i}$, let $g_{i, j}$ denote the rank that the distance between $\mathbf{x}_{i}$ and $\mathbf{x}_{j}$ takes in a sorted ascending list of distances between $\mathbf{x}_{i}$ and all $\mathbf{x}_{j \neq i}$. The $Y$-conditioned mean rank is then $G_{i}^{k}(X \mid Y)=\frac{1}{k} \sum_{j=1}^{k} g_{i, w_{i, j}}$, and we here define

$$
L(X \mid Y)=\frac{1}{N} \sum_{i=1}^{N} \frac{G_{i}(X)-G_{i}^{k}(X \mid Y)}{G_{i}(X)-G_{i}^{k}(X)} .
$$

where $G_{i}(X)=\frac{N}{2}$ and $G_{i}^{k}(X)=\frac{k+1}{2}$ denote the mean and minimal mean rank, respectively. The measures $S(Y \mid X), H(Y \mid X), M(Y \mid X)$, and $L(Y \mid X)$ are defined by exchanging the role of $X$ and $Y$ in the above definitions. We use the notation $A$ to refer to the group of all measures $S, H, M, L$.

A fundamental signature of non-synchronizing couplings $X \rightarrow Y$ is that close states of $Y$ are mapped to close states of $X$ with a probability increased compared to independent dynamics. This signature is quantified by the measures $A(X \mid Y)$ which increase with increasing coupling strength. However, an increased probability of the opposite mapping, i.e. close states of $X$ are mapped to close states of $Y$, also holds, although to a weaker degree. Therefore, for couplings $X \rightarrow Y$ also $A(Y \mid X)$ increase compared to values for uncoupled dynamics (e.g. [9]). Hence, $A(X \mid Y)$ by themselves are not specific for couplings $X \rightarrow Y$. Therefore, we follow e.g. [12, 18, 19] in defining $\Delta A=A(X \mid Y)-A(Y \mid X)$ and here test whether $\Delta A>0$ is a sensitive and specific criterion for non-synchronizing couplings $X \rightarrow Y$.

As stated above, to use controlled conditions, we analyze uncoupled as well as unidirectionally coupled non-identical Lorenz dynamics superimposed with different types of noise. The Lorenz dynamics $X: \dot{v}_{1}=10\left(v_{2}-v_{1}\right)$, $\dot{v}_{2}=$ $39 v_{1}-v_{2}-v_{1} v_{3}, \dot{v}_{3}=v_{1} v_{2}-\frac{8}{3} v_{3}, Y: \dot{w}_{1}=10\left(w_{2}-w_{1}\right)+\varepsilon\left(v_{1}-w_{1}\right), \dot{w}_{2}=35 w_{1}-w_{2}-w_{1} w_{3}, \dot{w}_{3}=w_{1} w_{2}-\frac{8}{3} w_{3}$ was integrated using a 4-th order Runge-Kutta algorithm with fixed step size of 0.005 and a sampling interval of 0.03 time units, which corresponds to approximately 20 samples per cycle. We used random initial conditions and applied $10^{6}$ pre-iterations to diminish transients. As deterministic time series we use $\tilde{x}_{i}=v_{1}\left(t_{i}\right)$ and $\tilde{y}_{i}=w_{1}\left(t_{i}\right)$ and an autoregressive processes as noise time series: $\xi_{i+1}^{X, Y}=a^{X, Y} \xi_{i}^{X, Y}+\zeta_{i+1}^{X, Y}$. Here $\zeta_{i}^{X, Y}$ denotes uncorrelated Gaussian noise with zero mean and unit variance. All examples studied here can then be written in the general form: $x_{i}=d^{X} \tilde{x}_{i}+n^{X} \xi_{i}^{X}, y_{i}=d^{Y} \tilde{y}_{i}+n^{Y} \xi_{i}^{Y}$ for $i=1, \ldots N=2048$. Throughout all simulations we use fixed values of $k=5$, and $W=50$ and set $m$ and $\tau$ as specified below.

\section{RESULTS}

As a first example we use Lorenz dynamics superimposed with Gaussian uncorrelated noise $\left(d^{X, Y}=1, a^{X, Y}=0\right.$, Fig. 1). Apart from uncoupled dynamics $(\varepsilon=0)$, we study coupled dynamics with $\varepsilon$ separated by factors of 1.05 between 0.05 and 18. Within this set of coupling strengths, $\varepsilon_{G S}=9.28$ is the lowest value for which generalized synchronization is attained, as determined from the identical synchronization of two replicas of the driven $Y$ dynamics started at different initial conditions [20]. Apart from noise-free dynamics we use the noise amplitudes $I^{X, Y}=$ $\left[0.125 \times 1.5^{n}\right] \sigma^{X, Y}$ for $n=0, \ldots 12$, where $\sigma^{X, Y}$ denote the standard deviation of $\tilde{x}_{i}$ and $\tilde{y}_{i}$, respectively. The noise is superimposed either only to the driver: $n^{X} \in I^{X}, n^{Y}=0$; to the driver and to the response: $n^{X} \in I^{X}, n^{Y} \in I^{Y}$; or only to the response: $n^{X}=0, n^{Y} \in I^{Y}$.

For the noise-free dynamics the coupling direction is correctly detected by $\Delta A>0$ to a different degree for for $H$, $M$, and $L$ (Fig. 1). For asymmetric noise levels some biases occur resulting in $\Delta A<0$, i.e. the detection of the wrong coupling direction, for some range of $\varepsilon$. Importantly, comparing $H, M$, and $L$, the rank-based measure $L$ proposed here is least affected by asymmetric noise. For the measure $S$, a more complicated picture is obtained which allows us to discuss in detail the various sources of biases in the following. 


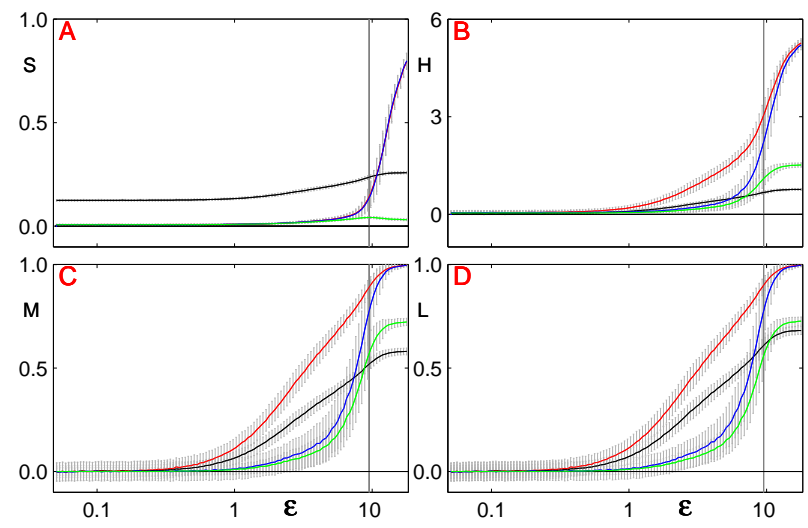

FIG. 1: (Color) Dependence of $A$ on $\varepsilon$ for coupled Lorenz dynamics $(m=8, \tau=4)$. For the noise-free case with $A(X \mid Y)$ in red and $A(Y \mid X)$ in blue. For $n^{X}=0.95 \sigma^{X}, n^{Y}=0$ with $A(X \mid Y)$ in black and $A(Y \mid X)$ in green. In panel A the blue curve almost covers the red one. Error bars depict \pm one standard deviation. Vertical lines mark $\varepsilon_{\mathrm{GS}}$.
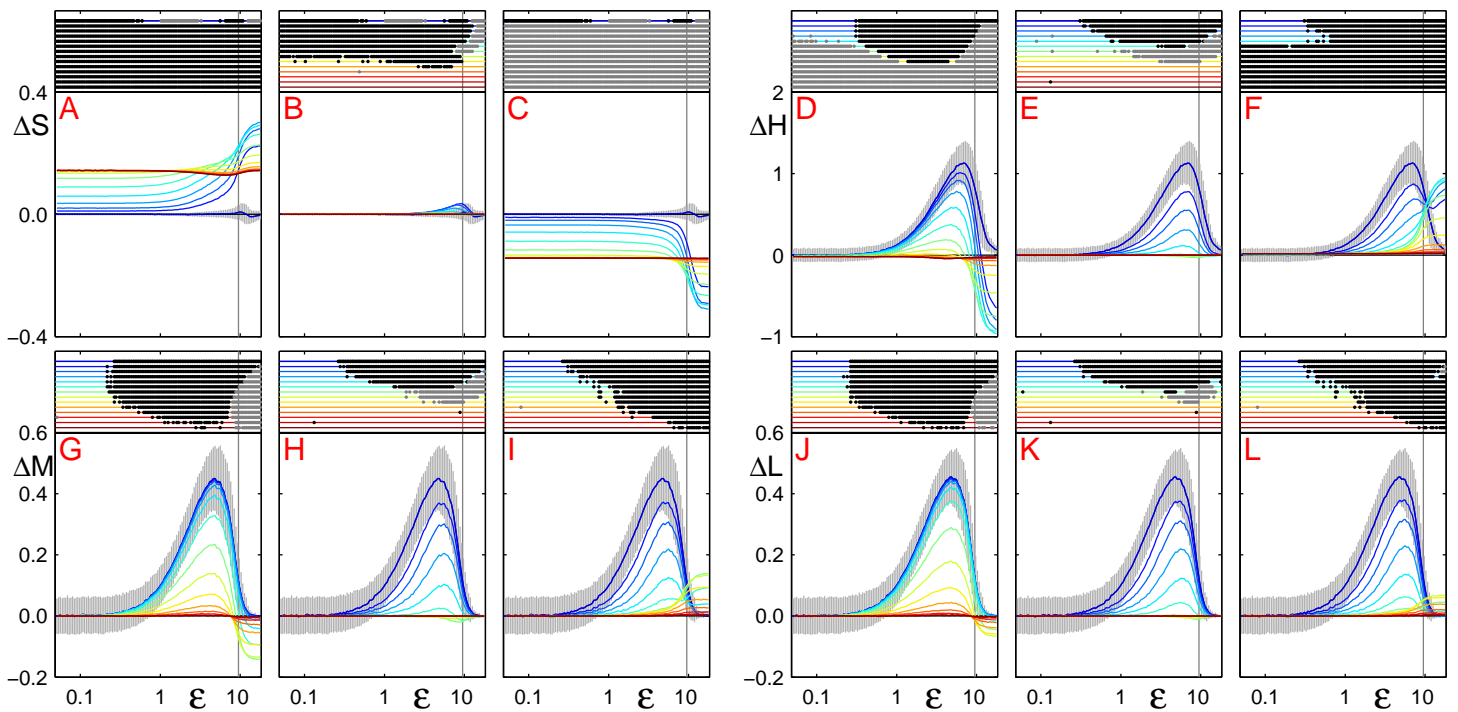

FIG. 2: (Color) Dependence of $\Delta A$ on $\varepsilon$ for $m=8, \tau=4$ for coupled Lorenz dynamics superimposed with noise. We used 1000 independent realizations of $\tilde{x}_{i}$ and $\tilde{y}_{i}$ for each $\varepsilon$ and added independent realizations of $\xi_{i}^{X}$ and $\xi_{i}^{Y}$ for each noise amplitude specified in the text (A,D,G,J: noise on $X ; \mathrm{B}, \mathrm{E}, \mathrm{H}, \mathrm{K}$ : noise on $X$ and $Y$; C,F,I,L: noise on $Y$ ). From blue to red colors indicate increasing noise levels. Error bars depict \pm one standard deviation and are shown for $n^{X, Y}=0$ only. Black and gray dots indicate significantly positive and negative $\Delta A$ values, respectively (Wilcoxon signed rank test at $\mathrm{p}=0.001$.). Vertical lines mark $\varepsilon_{\mathrm{GS}}$.

It was already indicated by [8] that the effective dimension $D_{X}$ is reflected in the distances defined above as: $\mathrm{E}\left[R_{i}^{k}(X) / R_{i}(X)\right] \sim(k / N)^{2 / D_{X}}$ and analogously for $Y$. Here $\mathrm{E}[\cdot]$ denotes the expected value across independent realizations of the dynamics. Since generally for zero and very small couplings $\mathrm{E}\left[R_{i}^{k}(X \mid Y)\right]=\mathrm{E} R_{i}(X)$, in this range of $\varepsilon$ we have $S(X \mid Y) \sim(k / N)^{2 / D_{X}}$, assuming ergodicity. Hence, $\mathrm{E}[\Delta S]>0$ for $D_{X}>D_{Y}$. Therefore, generally $\Delta S$ is nonzero even for uncoupled noise-free deterministic dynamics with slightly different effective dimensions. Here and below we simplify the notation $\mathrm{E}[\Delta A]$ to $\Delta A$ and apply the Wilcoxon test to determine whether nonzero values of $\Delta A$ are significant (See black and gray dots in Figs. 2 and 3). For the given Lorenz dynamics we have $D_{X}>D_{Y}$ at $\varepsilon=0$ and accordingly get $\Delta S>0$ (Figs. 1A, 2A-C). However, to be specific for couplings we should get $\Delta A=0$ for such independent processes. Upon increasing of $\varepsilon$ for $n^{X, Y}=0, D_{Y}$ at first increases due to the incorporation of the driver's degrees of freedom, and later decreases towards and beyond $\varepsilon_{\mathrm{GS}}$ due to the collapse of the joint dynamics to the synchronization manifold [9]. For the given dynamics this local maximum of $D_{Y}$ is reflected in $\Delta S<0$ found for an intermediate $\varepsilon$ range. Only for a narrow $\varepsilon$ range close to $\varepsilon_{\mathrm{GS}}$ the stronger mapping of close states in $Y$ to close states in $X$ leads to $\Delta S>0$. Upon increasing of the noise level the effective dimension converges to the embedding dimension $m$. Therefore, for asymmetric noise levels of $X$ and $Y$ the difference in $D_{X}$ and $D_{Y}$ and thereby $\Delta S$ 

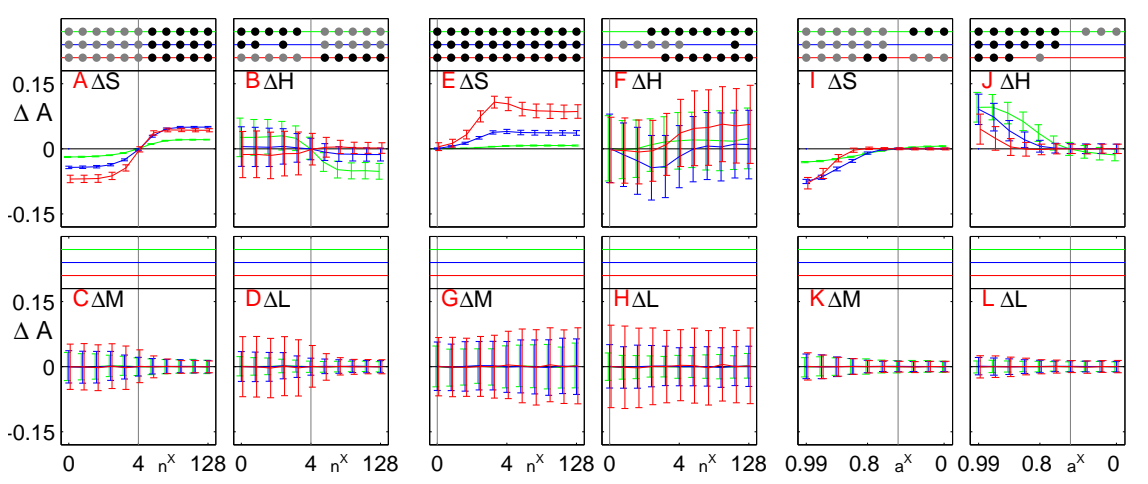

FIG. 3: (Color) Analogous to Fig. 2 but for independent dynamics. Green: $m=4, \tau=1$; blue: $m=6, \tau=4$; red: $m=8, \tau=12$. (A-D) Uncoupled Lorenz dynamics superimposed with asymmetric levels of white noise in dependence on $n^{X}$. Here vertical lines mark $n^{Y}$. Slight offsets on the abscissa are used to distinguish different error bars. (E-H) Same as (A-D) but here for uncoupled Lorenz dynamics with asymmetric levels of Gaussian autocorrelated noise. (I-L) Same as (A-D) but for purely stochastic time series with asymmetric autocorrelation strengths in dependence on $a^{X}$. Here vertical lines mark $a^{Y}$.

is dominated by this asymmetry. Even for symmetric noise levels $n^{X}=n^{Y}$ measured relative to the respective standard deviation of $X$ and $Y$, the impact of this noise can be asymmetric depending on the fine structure of the dynamics. A further source of bias, that $S$ shares with $H$, is its nonlinear dependence on the conditioned distances. (More specifically the asymmetry of this nonlinearity around $\mathrm{E}\left[R_{i}^{k}(X \mid Y)\right]$ is problematic cf. [12]). The impact of the nonlinearity depends on the variance of $R_{i}^{k}(X \mid Y)$ which in turn depends in a non-trivial way on intrinsic properties of $X$ and $Y$, the relative noise amplitude, noise color, and the parameters $m, \tau$, and $k$. Hence, this nonlinearity further contributes to $\Delta S \neq 0$ for independent but asymmetric dynamics (Figs. 1A, 2A-C).

For uncoupled noise-free Lorenz dynamics we get $\Delta H=0$ (Figs. 1B, 2D-F). With increasing coupling strength the stronger mapping of close states in $Y$ to close states in $X$ leads to $\Delta H>0$. Therefore, at first sight $\Delta H>0$ seems sensitive to and specific for couplings $X \rightarrow Y$. However, at zero and low $\varepsilon$ and for asymmetric noise levels the nonlinear dependence on the conditioned distances leads to $\Delta H>0$ for $n_{X}>n_{Y}$, and $\Delta H<0$ for $n_{X}<n_{Y}$. Furthermore, since $\mathrm{E}\left[R_{i}^{k}(X \mid Y)\right]=\mathrm{E} R_{i}^{k}(X)$ for $\varepsilon$ close to $\varepsilon_{G S}$ a term proportional to $(k / N)^{D_{X} / 2}$ contributes to $H(X \mid Y)$. In consequence for $D_{Y}>D_{X}$ the value of $\Delta H$ is increased. For symmetric noise levels, this supports the detection of the correct coupling direction because $D_{Y}>D_{X}$ due to the coupling. Asymmetric noise levels, however, bias $\Delta H$ at high $\varepsilon$. Nevertheless, for moderate noise levels $H$ is more robust for the detection of weak directional couplings than $S$ [9] (Figs. 1B, 2D-F).

Therefore, as already indicated by [9], the value of $\Delta S$ and $\Delta H$ not only reflect the mapping of close states in one dynamics to close states in the other but also the interplay of different biases. Before examining the impact of these biases on $M$ and $L$ we address a problem shared by the measures $S, H, M$, and $L$ that does not express any bias. Rather this problem is not only affecting state space based measures but shared by the above mentioned other coupling direction detection techniques applied to strongly coupled dynamics. Here this problem leads to the following effect: Regarding intermediate symmetric noise levels for strong couplings we find $\Delta A<0$ for all measures (Fig. 2B,E,H,K). Here both $A(X \mid Y)$ and $A(Y \mid X)$ attain high values, and $\Delta A$ can have different signs or vanish depending on the dynamics making a reliable detection of a coupling direction impossible (Fig. 1). Furthermore, even symmetric noise levels in general have different impacts on the mappings of close states in the one dynamics to close states in the other, due to their different local slopes. The mappings' geometry and thereby $A(X \mid Y)$ and $A(Y \mid X)$ as well as their noise robustness depend on the specific dynamical system. We should note that $L$ is least affected by this problem.

For independent dynamics generally the expected values of $M(X \mid Y), M(Y \mid X)$ and thereby the one of $\Delta M$ are all zero (Figs. 1C, 2G-I) [26]. This is due to the linear dependence on the conditioned distances $R_{i}^{k}(X \mid Y)$, and any bias caused by the nonlinearities described above for $S$ and $H$ does not affect $M$. Nonetheless, factorizing the term $R_{i}(X)$ in $M(X \mid Y)$, we see that the slope of this linear dependence is determined by the denominator $\left(R_{i}(X)\left(a(k / N)^{2 / D_{X}}-1\right)\right)$, where $a$ is some unknown factor. Therefore, for zero coupling the across realization variance of $\Delta M$ depends on $D_{X}$ and $D_{Y}$ while the mean remains unaffected and zero (This will be appreciated better in Fig. 3.). For $\varepsilon$ close to $\varepsilon_{G S}$, also the numerator of $M(X \mid Y)$ tends to $1-\left(b(k / N)^{2 / D_{X}}\right)$. Here $b$ is different from $a$ and depends on $\varepsilon$. Given that, for $\varepsilon$ close to $\varepsilon_{G S}$ the measure $M(X \mid Y)$ behaves similar to $H(X \mid Y)$ in that lower values are obtained for a higher $D_{X}$ though this bias is less pronounced for $M$ (Fig. 1C, 2G-I).

All advantages gained by virtue of the appropriate normalization of $M$ are inherited by $L$. The remaining dependence on the dimensionality for $\varepsilon$ close to $\varepsilon_{G S}$ is diminished since in contrast to distributions of distances, distributions of ranks of distances are always uniform. Notice that although this bias is still significant for a smaller region of 
asymmetric noise levels and coupling strengths, its absolute value is substantially lower for $L$ than for $M$ (Fig. 1D, 2J-L). As mentioned above for $\varepsilon$ close to $\varepsilon_{G S}$ a reliable coupling direction detection is impossible since $M(X \mid Y)$, $M(Y \mid X)$ and $L(X \mid Y), L(Y \mid X)$ are all close to one. However, for noisy dynamics these quantities decrease hiding the unreliability of the coupling direction. This decrease is less asymmetric for $L$ than for $M$, representing an important advantage of $L$.

To further study the specificity of $\Delta A>0$ for couplings $X \rightarrow Y$ and the influence of the embedding parameters, we now focus on independent dynamics. At first we consider two examples of uncoupled Lorenz dynamics superimposed with asymmetric noise levels. While both examples share $\varepsilon=0, d^{X, Y}=1, a^{Y}=0$, and $n^{X}=[0,0.25,0.5,1, \ldots, 128]$, we have $a^{X}=0, n^{Y}=4$ in the first and $a^{X}=0.97, n^{Y}=0$ in the second example. Here $n^{X, Y}$ are given as unitless amplitudes not related to the standard deviation of $\tilde{x}_{i}$ and $\tilde{y}_{i}$. Again nonzero values for $\Delta H$ and $\Delta S$ are obtained for these independent dynamics. Due to the dominance of the bias related to asymmetric effective dimensions the sign of $\Delta S$ is independent of the embedding parameters (Fig. 3A,E). In contrast, for $H$ the only bias for uncoupled dynamics is the nonlinear dependence on $R_{i}^{k}(X \mid Y)$. The variance of $R_{i}^{k}(X \mid Y)$ and thereby the sign of $\Delta H$ depend on the embedding parameters and asymmetry of the noise amplitudes (Fig. 3B,F). In contrast, the mean values of $\Delta M$ and $\Delta L$ are never significantly different from zero. Only their standard deviation is influenced by the relative noise levels and $m$ and $\tau$. In consequence, not a single false positive coupling detection is obtained by $M$ and $L$ (Fig. 3C,D,G,H).

As last example we use purely stochastic time series with asymmetric degrees of autocorrelation $d^{X, Y}=0, a^{Y}=0.5$, $n^{X, Y}=1$, and $a^{X}=[0.99,0.98,0.95,0.9,0.8,0.65,0.5,0.35,0.2,0]$. For lower values of the embedding window $(m-1) \tau$ a stronger autocorrelation is reflected in a lower effective dimension. Accordingly $\Delta S>0$ and $\Delta H<0$ for $a^{X}<a^{Y}$ and viceversa for $a^{Y}<a^{X}$ (Fig. 3I,J and cf. [21]). For high embedding windows and in particular for high $\tau$ the impact of short correlations is reduced since the state space is filled more homogeneously. In consequence, the biases of $S$ and $H$ due to the nonlinear dependence on the conditioned distances become more relevant resulting in different signs of $\Delta S$ and $\Delta H$. Like for the two previous examples $\Delta M$ and $\Delta L$ exhibit not a single false positive coupling detection (Fig. 3K,L).

\section{CONCLUSIONS}

We here proposed a rank-based measure for the estimation of directional couplings which is robust against asymmetries in the dynamics' structure or noise level. In particular, considerations about the dimensionality of nonlinear dynamics or the effect of noise on local densities can also be valuable to study approaches such as transfer entropy or non-linear Granger causality, since they are also based on local estimations of the dynamics' geometry. For the measures considered here, by virtue of their common more appropriate normalization, the measures $\Delta M$ and $\Delta L$ are clearly more sensitive and specific for directional couplings than $\Delta H$ and $\Delta S$. Furthermore, the use of rank statistics endows $\Delta L$ with a higher robustness as compared to $\Delta M$.

Rank transformations were previously used for the calculation of other measures of interdependence, for example the mutual information [22, 23]. However, for these techniques a global rank transformation of the time series is performed. This is conceptually different from our approach since we use local ranks of distances of spatially nearest neighbors of individual embedding vectors in reconstructed state spaces.

We here illustrate our findings using a thorough statistical assessment of coupled and uncoupled Lorenz dynamics as a representative example. Analogous results were obtained for other model systems. Therefore, our rank-based measure $L$ promises to reliably detect directional couplings also from experimental signals. This holds in particular when asymmetries in the dynamics' structure, noise color, or noise level might be present. It is important to keep in mind, however, that in application to experimental data other types of biases that were not considered here can affect any coupling detection technique. In particular, the assumption that the dynamics are at least approximately stationary can be violated. Furthermore spurious detections of interdependence can be caused by mixing of independent sources through the recording process. Therefore, the degree to which the improvements shown here for the rank-based measure $L$ result in a substantial improvement in the detection of meaningful directional couplings from experimental data remains subject to future work.

\section{Acknowledgments}

DC was supported by the grant 2008FI-B 00460 of the 'Generalitat de Catalunya' and European Social Funds. RGA acknowledges grant BFU2007-61710 of the Spanish Ministry of Education and Science. We are grateful to A. 
Ledberg for suggesting to test the use of ranks.

[1] C. W. J. Granger, Econometrica 37, 424-438 (1969).

[2] R. Dahlhaus, Metrika 51, 157-172 (2000).

[3] B. Schelter, and M. Winterhalder, and M. Eichler, and M. Peifer, and B. Hellwig, and B. Gushlbauer, and C. H. Lüking, and R. Dahlhaus, and J. Timmer, J. Neurosci. Meth. 152, 210-219 (2005).

[4] E. Pereda, R. Q. Quiroga, and J. Bhattacharya, Progress in Neurobiology 77, 1-37 (2005).

[5] Y. H. Chen, G. Rangarajan, and J. F. Feng, and M. Z. Ding, Phys. Lett A 324, 26-35 (2004).

[6] T. Schreiber, Phys. Rev. Lett. 85, 461-464 (2000).

[7] M. G. Rosenblum and A. S. Pikovsky, Phys. Rev. E. 64, 045202(R) (2001).

[8] J. Arnhold, P. Grassberger, and K Lehnertz, and C. E. Elger, Physica D 134, 419-430 (1999).

[9] R. Quian Quiroga, J. Arnhold, and P. Grassberger, Phys. Rev. E 61, 5142-5148 (2000).

[10] D. A. Smirnov and B. P. Bezruchko, Phys. Rev. E 68, 046209 (2003).

[11] H. Nalatore, M. Ding, and G. Rangarajan, Phys. Rev. E 75, 031123 (2007).

[12] R. G. Andrzejak, A. Ledberg, and G. Deco, New J Phys 8, 6 (2006).

[13] A. Cenys, G. Lasiene, and K. Pyragas, Physica D 52, 332 (1991).

[14] S. J. Schiff, P. So, and T. Chang, and R. E. Burke, and T. Sauer, Phys. Rev. E 54, 6708 (1996).

[15] R. Quian Quiroga, A. Kraskov, and T. Kreuz, and P. Grassberger, Phys. Rev. E 65, 041903 (2002).

[16] H. Kantz and T. Schreiber, Nonlinear Time Series Analysis (Cambridge Univ. Press, Cambridge, UK, 2003).

[17] R. G. Andrzejak, A. Kraskov, and H. Stögbauer, and F. Mormann, and T. Kreuz, Phys. Rev. E. 68, 066202 (2003).

[18] M. C. Romano, M. Thiel, and J. Kurths, and C. Grebogi, Phys. Rev. E 76, 036211 (2007).

[19] D. A. Smirnov and R. G. Andrzejak, Phys. Rev. E 71, 036207 (2005).

[20] L. Kocarev and U. Parlitz, Phys. Rev. Lett. 76, 1816 (1996).

[21] C. J. Stam and B. W. van Dijk, Physica D 163, 236 (2002).

[22] A. M. Fraser and H. L. Swinney, Phys. Rev. A. 33, 1134 (1986).

[23] C. J. Cellucci, A. M. Albano, and P. E. Rapp, Phys. Rev. E 71, 066208 (2005).

[24] Due to the exclusion of $W$ neighbors, $N$ has to be adjusted, and cases $i<W, N-i<W$ need to be further distinguished. To simplify the notation we write all formulas for $W=0$.

[25] See EPAPS Document No. 1 for source code of all the measures.

[26] An exception occurs for strong periodic components in $X$ or $Y$, since in this case $\mathrm{E}\left[R_{i}^{k}(X \mid Y)\right]=\mathrm{E} R_{i}(X)$ does not necessarily hold. 